\title{
Philosophiques
}

\section{Nouvel essai sur les sandales d'Empédocle}

\section{Marc Renault}

Volume 3, numéro 1, avril 1976

URI : https://id.erudit.org/iderudit/203042ar

DOI : https://doi.org/10.7202/203042ar

Aller au sommaire du numéro

Éditeur(s)

Société de philosophie du Québec

ISSN

0316-2923 (imprimé)

1492-1391 (numérique)

Découvrir la revue

Citer cet article

Renault, M. (1976). Nouvel essai sur les sandales d’Empédocle. Philosophiques, 3(1), 33-54. https://doi.org/10.7202/203042ar d'utilisation que vous pouvez consulter en ligne.

https://apropos.erudit.org/fr/usagers/politique-dutilisation/ 


\title{
NOUVEL ESSAI SUR LES SANDALES D'EMPÉDOCLE
}

\author{
par Marc Renault
}

Même dans la subversion révolutionnaire, les changements de systèmes politique, scientifique, moral et religieux ne sont jamais une liquidation complète du passé. L'accélération des mutations culturelles ne supprime pas le fait que les générations successives héritent d'une tradition et n'ont pas à repartir à zéro pour inventer leur langage et négocier leurs formes de vie collective. L'éducation et l'enseignement ne peuvent donc se définir ni par la seule fonction critique, ni comme la seule fomentation de la créativité, et doivent commencer par transmettre simplement une tradition. Descartes s'en plaignait, déplorant qu'on doive être enfant avant que d'être homme, mais il conseillait aussi à des amis d'envoyer leurs fils à Laflèche, en vantant la qualité de l'instruction qu'il y avait reçue. La ruche à cadre augmente le rendement en dispensant les travailleuses de toujours recommencer la cimentation de tout le réseau de cire. Le temps gagné sur la construction des alvéoles est consacré à la production du miel. Or «le miel est seul nutritif: la fin de toute l'opération " ${ }^{1}$. Ainsi, nous n'avons pas chacun à recommencer l'invention de notre humanité, mais toute cette cire ne sert à rien si nous n'arrivons pas à y mettre notre miel.

Cette tension entre l'autorité de la tradition, d'une part, et l'esprit d'invention et de critique, d'autre part, se retrouve dans tous les domaines de la culture, même dans la science. Pour être en mesure de fournir une contribution à la science, il faut un apprentissage. Plus la science en question est développée, plus cet apprentissage fera appel à l'autorité. Dans la physique, par exemple, il est impossible à l'apprenti de tout reprendre et de reconstruire à neuf lui-même ce qu'il apprend. Il doit faire

1. BLONDEL, Maurice, Carnets intimes, tome 2 (1894-1949), Paris, Cerf, 1966, D. 185. 
confiance à ceux qui ont découvert et qui ont su. On se trouve ici devant une espèce de croyance dans la communauté des savants qui ont constitué la physique.

D'un autre côté, à l'intérieur de son propre domaine spécialisé de recherche, là où sa compétence lui permet d'apporter du nouveau à sa discipline, le physicien peut en arriver à contester la science officielle et proposer des réformes. Il n'est d'ailleurs intéressé à cette transformation du système du savoir qu'en raison de l'autorité de ce savoir sur lui et parce qu'il s'en fait une valeur. Mais si le chercheur en train de tester une hypothèse a l'intention de mettre en doute certains aspects de la science, il reste prêt à abandonner la chose sur manque de vérification. Ainsi l'hypothèse de recherche est-elle comme suspendue entre un peut-être bien que oui et un peut-être bien que non, mais cette suspension n'affecte pas la valeur accordée au système de la physique. Même des renversements comme la physique quantique et la relativité se sont faits à l'intérieur d'une confiance envers la tradition de la physico-mathćmatique.

Mais au moment où Einstein s'étonne qu'il puisse y avoir une correspondance entre le contenu a posteriori de l'expérience et les constructions a priori de la mathématique, il ne pense plus en physicien, mais en philosophe. La crise de confiance envers la physique n'est pas un problème de physique. Si on me permet un raccourci, je dirais 'qu'il s'agit d'un affrontement entre la liberté et la civilisation, cette dernière étant comprise au sens kantien comme le résidu factuel de ce qui a d'abord été une création ou invention culturelle.

Max Scheler a décrit la culture comme un «façonnement» de soi, de la société et du monde à partir d'une finalité idéale. Le point de rupture entre nature et culture est précisément la liberté, principe du façonnement. «Le monde s'est développé au point de produire réellement l'homms à son sommet, l'homme doit à son tour s'élever jusqu'à former en lui-même le monde, idéalement ${ }^{2}$. Aux mots réellement et idéalement, on pourrait substituer ceux de naturellement et étbiquement, car la notion de culture n'a pas de contenu spécifique par rapport aux détermi-

2. SCHELER, Max., L'bomme et l'bistoire, traduction par M. Dupuy, Paris, Aubicr, 1955 , p. 105. 
nismes naturels si on lui enlève l'ingrédient de la raison pratique, ou liberté kantienne. Il y a contresens, à ce point de vue, à vouloir opposer culture et liberté. Mais dans la mesure où les créations culturelles deviennent un simple bagage traditionnel de la collectivité, elles peuvent survivre détachées de leur origine éthique et se transmettre aux héritiers comme des contraintes sans « raison». Les gestes, qui avaient été ceux de la libération dans le passé, exercent une préemption sur la conscience des descendants et deviennent au mieux de simples conventions ct conformismes, au pire des structures carrément opprimantes. Descartes rêvait d'inventions mécaniques pour libérer les hommes des labeurs asservissants. La révolution industrielle passe, et voici que nous rêvons de nous libérer de l'asservissement à la machine.

Tout héritage culturel transmis à travers le temps, sous forme de produits de civilisation, est susceptible de passer par une suspension de sa validité. Lorsque cette possibilité de suspension est manifeste comme pouvant prendre une extension universelle, l'entreprise philosophique devient consciente de sa spécificité. L'histoire de la philosophie peut se concevoir comme histoire des crises de confiance, ou crises de crédibilité envers une tradition. "Jamais nous ne sommes dans l'abstrait de l'in" telligence ou de la volonté nues. Toujours nous sommes engagés dans la tradition humaine ou divine qui nous a amenés au point où nous sommes ${ }^{3}$. Dès lors, le principal intérêt de l'histoire de la philosophie serait de nous mettre sous les yeux ce perpétuel. rebondissement de la pensće personnelle sur sa situation traditionnelle et la nécessité d'une reconstruction qui ajuste la réalité aux convenances de... de quoi?

Si nous suivons la suggestion de notre tradition occidentale, les convenances sont ici celles de la raison. Et cela constitue déjà un problème. Parler de raison, cela implique référence à quelque chose de transcendant aux situations historiques singulières des philosophes, alors que la rupture du lien avec la tradition tient à l'histoire même de chaque philosophe. « La conscience et la réflexion, dans l'individu comme dans la société, naissent d'un trouble, d'un contraste, d'un désaccord. Ainsi

3. BLONDEL, op. cit., p. 75 . 
la souffrance est nécessaire pour nous tirer de l'insensibilité et nous rendre le goût de nous-même: sans elle nous restons sans saveur ${ }^{4}$. Chacun doit recommencer la philosophie à partir de sa propre «disconvenance». En termes hégéliens, on pourrait dire que la confiance s'aperçoit qu'elle n'est pas savoir, mais simple croyance. En devenant croyance pour soi, c'est-à-dire croyance consciente de soi comme croyance, elle acquiert la possibilité de passer à la non-croyance. Me savoir croyant, c'est apercevoir d'un point de vue rationnel que la croyance est une détermination de ma subjectivité. Dès lors le corrélat de ma confiance se dissipe dans l'incertitude. Tour à tour je suis ferme et décidé lorsque, simplement, je crois et fais confiance pragmatiquement, et je suis hésitant et incertain lorsque je pense réflexivement ma foi. Dans les mots de Sartre: «La croyance est un être qui se met en question dans son propre être, qui ne peut se réaliser que dans sa destruction, qui ne peut se manifester à soi qu'en se niant; c'est un être pour qui être, c'est paraître, et paraitre, c'est se nier. Croire, c'est ne pas croire». Un peu plus loin dans la même page de L'être et le néant, il ajoute, «je ne pourrai me dissimuler que je crois pour ne pas croire et que je ne crois pas pour croire ${ }^{5}$. Est-ce que Sartre était conscient de répéter ici les formules de saint Augustin, de saint Anselme, de Richard de Sî-Victor? Au-dessus des changements historiques et des différences de situation, n'y a-t-il pas plutôt simplement une loi de la pensée humaine, à savoir qu'aussitôt qu'il se comprend comme faisant confiance à une tradition, le penseur ne peut manquer de vouloir dépasser cet état pour voir les choses par lui-même. Dans les termes de saint Anselme, credo mt intelligan. La foi n'est justifiable que si elle est sur la voie de l'intellection. Pour le théologien médiéval, la foi n'échappe à l'absurdité que si on la considère dans son rapport à la vision. Ayant compris cela, le croyant chrétien voit aussi qu'il peut continuer d'être croyant seulement s'il espère la vision. 'Tout se ramène à la question de l'espérance ou du désespoir.

En décrivant l'émergence de la philosophie comme une crise de confiance, il est donc utile d'ajouter que cette crise se dénoue

4. BLONDEL, Maurice, Carnets intimes, tome 1 (1883-1894), Paris, Cerf, 1961, p. 313.

5. L'Être et le néant, p. 110. 
sur le fond des espérances du philosophe. Aucune crise ne pourrait toucher l'homme sans espérance. Mais tant qu'il y a de la vie, il y a de l'espoir, dit le proverbe. Et tant qu'il y a de l'espoir, il y a de la croyance en ce que l'avenir peut donner, il y a une ontologie, qui est aussi une métaphysique, assiégée sans. doute et en butte au soupçon, mais bien en place.

La crise philosophique n'est possible qu'à partir d'un système espérance-foi porteur d'une ontologie. Le questionnement se présente sans doute en termes d'intérêt pour la vérité et veut examiner le bien-fondé des affirmations dans lesquelles la croyance exprime ce qui est, mais à travers la suspension des jugements à portée ontologique, le questionnement vise en fin de compte ce que le sujet attend de l'existence et cherche à réaliser dans son agir. Les questions spéculatives ne sont ainsi jamais détachables des intérêts pratiques, sinon par des conventions méthodologiques. Or ces conventions tombent sitôt que le philosophe, cessant de jongler avec des possibles dans sa théorie, décide, veut, fait quelque chose. Le contenu spéculatif de la foi peut bien être l'objet premièrement touché par la crise, mais un tel objet ne vaut la peine d'être soumis à l'examen critique que s'il représente quelque chose pour le croyant et touche à son ontologie. Que la mythologie d'Homère soit balayée par la philosophie comme une représentation inadéquate de la religion, cela pouvait toucher les philosophes vivant dans le milieu où cette mythologie avait de l'autorité, mais pour nous qui n'avons jamais cru à l'Olympe, il y a là un simple exemple historique de ce que la philosophie peut faire par rapport à la culture dans laquelle elle s'exerce. Il y a une analogie entre la situation de Socrate dans son milieu et la situation du philosophe d'aujourd'hui dans son milieu.

Voici comment Descartes décrit le dualisme introduit par la crise. Avec une simplicité désarmante, il écrit, au début de la troisième partie du Discours: "Afin que je ne demeurasse point irrésolu en mes actions, pendant que la raison m’obligerait de l'être en mes jugements, et que je ne laissasse pas de vivre dès lors le plus heureusement que je pourrais, je me formai une morale par provision ${ }^{6}$. Descartes nous fait ici remarquer

6. Oentres, Plćiade, pp. 140-141. 
comment le questionnement, si radical qu'on le suppose, laisse intact un fond pragmatique où le jugement continue de s'exercer alors même qu'il a réflexivement «décidé» de tout suspendre. Au moment même où le questionnement grignote mes convictions et les rend révocables, je continue de juger et d'agir, tout comme si je savais et si j'étais certain. Ainsi la résolution pragmatique côtoie l'irrésolution réflexive au sein de la même existence et la possibilité même d'entretenir ce conflit manifeste, aux yeux de Descartes, la liberté de la pensée. C'est en effet, dans ses propres mots, «l'esprit usant de sa liberté ${ }^{7}$ qui introduit l'irrésolution du jugement. Cette liberté ne balaie pas la croyance, mais la suspend, dans l'attente d'une sentence de la raison. Pour pouvoir passer devant le tribunal de la critique, les croyances doivent paraitre comme peut-être révocables et donc d'une validité provisoire. Un citoyen n'est cité devant la cour qu'en raison d'un délit possible.

Descartes croyait-il vraiment récupérer ce qui resterait de son provisoire dans la certitude d'une philosophie bien établie et stabilisće? Le philosophe ne meurt-il pas plutôt avant d'avoir jamais vu le bout de cette dialectique entre la suspension cri. tique et ses croyances, de sorte que les options de fond sur lesquelles il joue sa vie débordent toujours sa philosophie? Celle-ci sert d'élucidation, d'évaluation, de purification, de réfutation ou de justification, mais non de fondement de la croyance. Et s'il arrive qu'un philosophe renverse certaines de ses propres croyances par voie critique, il se replie en même temps sur d'autres. Le nouvel état de la croyance qui pourrait sortir d'une crise réflexive sera provisoire à son tour au regard d'une sus. pension ultérieure, de sorte que l'histoire sanctionne et rend final ce qui était possiblement révocable jusqu'au dernier instant.

Mais comment y a-t-il un «révocable», et de quoi est-il fait? Dans son ouvrage sur l'évolution de la théorie déductive, Ortega y Gasset fait remonter l'institution de la philosophie à cette situation où des hommes sont exposés à des croyances divergentes et commencent à se demander laquelle est digne de créance. Le besoin de trancher, au mérite, entre des croyances, c'est le problème de la vérité. Celui qui vit pleinement et pure-

7. Abrégé des míditations, dans Oeưres, Pléiade, p. 262. 
ment de la croyance n'a pas de problème au sujet de la vérité. Ortega y Gasset va jusqu'à dire qu'il lui est impossible de sentir ou de comprendre ce qu'on signifie par ce mot de vérité : "While one lives fully in belief, it is impossible to feel or even understand what sort of thing Truth is ${ }^{8}$. C'est dans une cité aux croyances disloquées que peut apparaitre Socrate, mais la crise de la sophistique nous semble, de loin, laisser la culture athénienne dans un état assez homogène, alors que la situation contemporaine présente un écheveau autrement complexe. Socrate pouvait assez facilement identifier la tradition devenue révocable, alors que nous avons déjà beaucoup à faire, dans la mosaïque pluraliste, pour identifier quelle est au juste la situation de confiance préphilosophique sur laquelle porte le questionnement. Et s'il est déjà difficile, pour chaque philosophe, de tirer au clair cette question pour soi-même, à plus forte raison aura-t-il de la peine à prévoir qui son discours pourrait intéresser, qui le trouverait pertinent. Platon, saint Thomas, Descartes, et même Kant savaient à qui ils s'adressaient, mais aujourd'hui on jette sa bouteille à la mer.

En cherchant à refaire un consensus autour d'un discours «désontologisé », où l'intentionalité s'aligne sur un nécessaire, qui est un simple possible ontologique, Husserl invite le philosophe à contourner l'ontologie antéprédicative. Sortie des fondrières de la croyance, la philosophie peut se constituer $A l s$ strenge $W$ issenschaft ${ }^{9}$ et rallier les esprits avec le même succès que la science. Pour obtenir ce ralliement, il est requis de « réduire » l'intention au sens pur, à l'essence. Il faut même purifier la logique de tout ce qui serait un code des normes de pensée, car il y a là l'ontologie cachée d'une pensée qui s'affirme d'abord elle-même comme pouvoir législateur. "L'intérêt normatif qui gagnait du terrain tendait, comme on le conçoit facilement, à masquer l'objectivité idéale des formations ellesmêmes ${ }^{10}$. L'objectivité intelligible est en effet masquée comme

8. The Idea of Principle in Leibniz and the Erolution of Deductive Theory, New York, W.W. Norton, 1971, p. 298.

9. Pbilosophie als strenge Wissenschaft (1910), Frankfurt, Klostermann, 1965.

10. HUSSERL, Edmond, Logique formelle et logique transcendantale, essai d'une critique de la raison_logique, traduit par Suzanne Bachelard, 2e éd., Paris, PUF, 1965, paragraphe 11, p. 63. 
fondement si on la reporte sur le fond d'une ontologie de la pensée.

$\bar{A}$ ne considérer que cette surface de la question, la phénoménologie invite au consensus scientifique par une disjonction entre l'essence et l'existence, entre l'analyse transcendantale des conditions de possibilité et les affirmations ontologiques, entre le discours théorique et l'espérance pratique. Il n'y a là rien à quoi s'objecter, tant que la réduction reste consciente de ce qu'elle est, à savoir une fiction méthodologique. Et Husserl le savait bien. L'emploi constant de l'Einklammerung, de la parenthèse empruntée au langage mathématique, indique bien la décision de ne pas tenir compie de ce qui, par ailleurs, ne se supprime pas ${ }^{11}$. La constitution phénoménologique des essences ne fonde pas une objectivité étrangère à celle de la conscience naïve. Son rôle n'est pas de remplacer mais de confirmer. La substitution d'un ego transcendantal de type kantien au cogito ontologique cartésien marque un progrès du questionnement, mais n'est en aucun cas une suppression de l'ontologie.

Dans des termes qui rappellent Fichte, Sartre nous montre un pour soi qui s'arrache à toute adhésion à l'être pour se constituer en aperception transcendantale. Mais dans ce mouvement de néantisation persiste une instance qui l'opère. Le pour soi peut certes monter la machine logique, mais il n'est pas vrai que cette décision puisse détacher la machine du fond qui la pose. Pour se débarrasser de soi-même, le pour soi devrait d'abord se mettre tout dans l'objet. «Ma propre transcendance ne peut que transcender », - (n'est pas pour soi-même un objet pouvant être transcendé), - « je la suis, je ne puis me servir d'elle pour la constituer comme transcendance transcendée ${ }^{12}$.

Autrement dit, le pour soi n'est ni représentable comme une donnée, ni objectivable par concept, ni réductible à la simple fonction logique du système des possibles, car à même la réduction méthodologique, il se pose comme l'ayant décidée. L'ego formel, postulé comme condition de l'unité du discours des essences nécessaires, est lui-même fondé sur l'ego ontologique

11. Voir les remarques de Quentin Lauer dans Pbenomenology; its Genesis and Prospect, New York, Harper and Row, 1965, pp. 88-90.

12. L'Être et le néant, p. 359. 
prenant sur soi de séparer sa fonction logique de ses croyances ontologiques. L'ordre des nécessités logiques est second et ne saurait se muer en philosophie première. Nous avouons cela tacitement toutes les fois que, du langage des nécessités, nous glissons à celui des possibles. En fait ce glissement s'est d'abord fait en sens inverse, du possible au nécessaire. Quant au possible lui-même, sa référence à l'être relève d'un jugement analytique. Il n'est donc pas inutile de revenir à Descartes.

Dans le doute cartésien, tout est révocable, sauf la certitude que c'est tout un de penser et d'être. Il n'y a pas moyen de suspendre complètement l'ontologie. On peut seulement décider de ne pas en tenir compte par méthode. Mais du sein même de l'entreprise critique où j'essaie de tout «fonder en raison», (i.e. : de ramener toute réalité à une logique où elle se manifeste comme pure possibilité nécessairement pensée), je sais que la critique représente en fin de compte l'intérêt d'une raison qui cherche par là à être. L'ontologie continue toujours d'affecter au moins le pouvoir de juger lui-même. L'ontologie précède ainsi le discours du savoir théorique et appartient à la foi antéprédicative. Il n'y a pas de milieu entre cette foi et le scepticisme. Il n'y a pas de confiance dans les possibilités de la pensée si celle-ci n'a pas d'abord confiance qu'elle est. Sur ce point, le cogito cartésien me semble tenir et le scepticisme de Hume constitue une antithèse frappante. On n'a pas à s'offusquer que le cogito soit traité de «mythe» sur le plan spéculatif ${ }^{13}$, car c'est de toute façon le mythe d'une réalité à laquelle on se confie ou ne se confie pas, indépendamment du savoir.

Kant dissocie les deux pôles du cogito. Comme condition du savoir, il est pure fonction logique. Comme position ontologique, il est liberté pratique. Le cogito qui affirme son être n'est pas un élément du discours de la science. Et cela est une difficulté dans l'interprétation du kantisme: où se trouve la soudure de l'usage spéculatif et de l'usage pratique de la raison? Fichte apparaît dans ce contexte. Il lui semble nécessaire de réunir dans un même Je, $I c b$, les deux usages et de se placer à un point de vue qui les transcende tous deux. D'une part, le cogito spéculatif s'isole dans les nécessités logiques, d'autre part, le

13. RYLE, Gilbert, The Concept of Mind, Harmondsworth, Peregrine Books, 1963, pp. 13-25. 
cogito pratique brise cet isolement et transgresse le savoir dans l'affirmation ontologique. N'y a-t-il pas une intuition originaire dont bifurquent les deux usages kantiens? Entre les objectivités nécessaires de la spéculation et les implications ontologiques de la pratique, n'y a-t-il pas le moyen terme de la possibilité? N'y a-t-il pas par conséquent un $J e$ qui transcende les deux usages et peut en conséquence les problématiser?

Descartes nous donne un cogito logique constitué à partir d'un cogito ontologique. Mais si on se pose la question du droit de l'ontologique à fonder le logique, on se retrouve avec un cogito logique s'essayant à fonder le cogito ontologique. Fichte demande la trève de cette dialectique. Dans son Essai d'un nouvel exposé de la doctrine de la science (1797), ${ }^{14}$, il formule l'axiome, implicite chez Descartes, que «je dois être pour penser», puis il demande: qui juge ainsi? La pensée sans doute. Il faudrait donc plutôt formuler ainsi: Je pense que je dois être pour penser. Postuler une condition ontologique de la pensée, c'est une exigence de pensée. $\bar{A}$ ce point de vue, c'est l'ontologie elle-même qui est seconde par rapport à son a priori dans la. pensée, et loin de pouvoir prendre appui sur une intuition ontologique au départ, je me vois dans l'obligation de justifier ma croyance à l'être à partir d'un $I c h$ faisant valoir des exigences au sujet de l'être. Le pouvoir de juger, qui énonce la nécessité d'être pour penser, est lui-même un acte de penser qui se considère tenu de se trouver un fondement. Il faut donc que l'intuition originaire contienne dans son unité la dichotomie dérivée entre la possibilité et l'être, entre le logique et l'ontologique, entre le théorique et le pratique. La postulation de l'être par la pensée et la fondation de la pensće par l'être se répondent à l'intérieur de l'unité originaire du Je.

L'idéalisme et le réalisme sont ainsi également possibles à l'origine, l'intelligence étant de sa nature l'unité de l'être et $\mathrm{du}$ voir, «Vereinigung des Seins, und des Sehens ${ }^{15}$. La différenciation de ces systèmes n'est imposée ni par les faits $a$ posteriori, ni par les nécessités spéculatives a priori. C'est pour-

14. Versuch einer neuen Darstellung der Wissenschaftslebre, édition de Fritz Medicus, Hamburg, Felix Meiner, 1954, p. 111.

15. Erste Einleitung in die Wisseschaftslebre, paragraphe 6 , édition Fritz Medicus, Hamburg, Felix Meiner, 1954, p. 22. 
quoi l'idéaliste ne peut réfuter le téaliste, ni celui-ci l'idéaliste. L'un et l'autre développent simplement des intérêts différentsà partir d'une même possibilité originaire qui est l'unité de la pensée et de l'être. Fichte marque ainsi un certain retour à Descartes, avec en plus la conscience que le discours philosophique est tout entier déterminé «durch Neigung und Interesse » ${ }^{16}$, par le penchant et par l'intérêt. L'ontologie que nous professons ne nous est pas imposée du dehors par des évidences qui contraignent le jugement, mais proposé au consentement à partir de cette indétermination première de l'intelligence où la pensée prescrit l'être et l'être prescrit la pensée dans une circularité originaire où-la simple logique ne peut par elle-même discerner lequel des termes fonde l'autre. Il y a ici quelque chose comme une antinomie kantienne spéculativement insoluble. «La raison ne livre aucun argument décisif en faveur de l'un ou de l'autre; en effet il ne s'agit pas de la liaison d'un terme dans une série...; il s'agit du début de la série, qui, en tant qu'acte absolument premier, ne dépend que de la liberté de la pensée, von der Freiheit des Denkens abhängt ${ }^{17}$. Fichte règle la question pour lui-même par une option résolument idéaliste. Lui objecte-t-on qu'une telle option manque de fondement dans l'expérience? Il répond fièrement: «La philosophie doit donner le fondement de toute expérience. Son objet se situe donc nécessairement en dehors de toute expérience, ihr Objekt liegt sonach notwendig ausser aller Erfahrung ${ }^{18}$. Fichte érige donc son option idéaliste en fondement et norme de l'expérience. Si la science se constitue ensuite dans les limites de l'expérience, sa nécessité et son objectivité seront des dérivés de la liberté: celle-ci n'est qu'en étant première. La science est un extrait de la moralité.

La critique kantienne avait soutenu qu'il n'y a de connaissance en général, donc de science, que de ce dont nous pouvons constituer a priori l'objectivité. Comme la liberté est l'a priori d'une telle constitution, comme elle opère cette séparation de la nécessité objective logique et de la position ontologique, elle-même n'est pas susceptible d'une conceptualisation

16. Ibidem, paragraphe 5, p. 19.

17. Loc. cit.

18. Op. cit., paragraphe 2, p. 11 . 
objectivante. D'autre part, si elle est pouvoir de séparation, elle l'est aussi de la réunion. Ce qu'elle a séparé dans la révocation de la confiance ontologique naive, elle le réunit forcément, mais non par le savoir. Nous avons affirmé plus haut qu'il n'y a pas de degré zéro de l'ontologie. Il faut ajouter que cette ontologie ne saurait être une science. Descartes s'est trompé en croyant que le sum de son cogito était un chaînon du savoit, mais il avait bien raison de penser que la science avait des racines métaphysiques, dans cette région où la penséeliberté, en sus de sa fonction d'objectivation cognoscitive, se concentre en certitude d'être, en attachement inconditionnel à l'être.

Une solution scientifique aux antinomies de la dialectique transcendantale impliquerait que nous disposons d'une ontologie scientifique. Kant nous a enlevé cette prétention. Mais avec Kant, Fichte pense que les antinomies sont de fait tranchées dans l'usage pratique, celui de la «réunion» où la liberté passe de la possibilité à la position effective. Dans le kantisme, ceci entraînait déjà un primat de la raison pratique dans l'ordre des affirmations métaphysiques. Kant était de plus convaincu de l'unité de la raison sous ses usages différents, mais restait embarrassé quant à la façon de penser cette unité. Fichte a moins de scrupules. La position première de la doctrine de la science, erster, schlechtbin unbedingter Grundsatz ${ }^{19}$, c'est le Je indivisible lui-même, à la fois être et principe. Ce Je est parce que je l'ai posé et je le pose parce qu'il est: Es setzt sich durch sein blosses Sein, und ist durch sein blosses Gesetztsein ${ }^{20}$. Ou encore, le Je qui pose et le Je posé ne diffèrent pas: Das sich setzende Ich, un das seinde Ich sind völlig gleich, Ein und ebendasselbe ${ }^{21}$. Fichte identifie ainsi le was ich bin (ce que je suis) au weil ich bin (parce que je suis). Et c'est à partir de cette unité primitive essence-existence qu'il peut y avoir un Je divisible, celui qui s'oppose un monde et en institue le savoir. De son indivisibilité principielle, et en elle, le Je tire une division dérivée dans laquelle un non-Je divisible est opposé à un Je

19. Grundlage der gesamten Wissenschaftslebre (1794), édition de Fritz Medicus, Hamburg, Felix Meiner, 1961, p. 11.

20. Ibidem, p. 17.

21. Ibidem, p. 18. 
divisible ${ }^{22}$. Or le Je de la science, (en termes kantiens la raison dans son usage spéculatif), est bien ce deuxième. La spéculation objectivante dérive ainsi d'une ontologie en s'en séparant, mais garde le stigmate de son origine en ceci que les nécessités du discours de science ne peuvent pas se dispenser d'être en même temps des possibilités de l'affirmation d'être. Encore une fois, il n'y a pas de degré zéro de l'ontologie. Mais aussi, l'ontologie ne saurait être une science.

Il ne faut donc pas compter sur une cessation prochaine du conflit des discours dans la république des philosophes. $\grave{\Lambda}$ partir des possibilités, souvent antinomiques, qui apparaissent dans la scission de l'objectivité, la «réunion » ontologique, si inévitable qu'elle soit en elle-même, n'a pas de guide spéculatif. Elle reflète les intérêts pratiques du philosophe et passe forcément par une option, une décision, un saut du possible à l'être. Fichte n'en fait pas mystère. "L'intérêt suprême et le fondement de tous les autres intérêts», dit-il, «est celui qui s'adresse à nous-mêmes. Il en est de même pour le philosophe. $\mathrm{Ne}$ pas perdre son Soi, sein Selbst, dans le raisonnement, mais l'affirmer et le maintenir, tel est l'intérêt qui guide invisiblement (unsichtbar) toute sa pensée ${ }^{23}$.

«Invisiblement», dit-il. Au gré de Fichte, cétait en effet l'inspiration invisible de Kant, mais cela est devenu visible avec la Wissenschaftslebre, la Doctrine de la science. La motivation secrète du kạntisme devient le principe explicite de l'idéalisme fichtéen. Parlant de lui-même à la 3 e personne, Fichte déclare: «L'auteur de la doctrine de la science présenta celle-ci en affirmant qu'elle s'accordait parfaitement avec la doctrine kantienne et riétait que cette dernière bien comprise ${ }^{24}$. La raison pour laquelle Fichte jette son dévolu sur le kantisme ainsi compris, c'est le Je autoaffirmatif, principe d'un savoir d'objets dont le développement ne peut en aucun cas revenir sur lui et le supprimer, c'est le Je qui ne consulte que son intérêt propre pour décider de ce à quoi il consentira comme être. Si le discours spéculatif n'est pas détachable de toute ontologie, il ne préjuge

22. Ich sctze im Ich dem teilbaren Ich ein teilbares Nicht-Ich entgegen. Ibidem, p. 30 .

23. Erste Einleitung. .., paragraphe 5, pp. 19-20.

24. Zueite Einleitung..., paragraphe 6, p. 55. 
cependant d'aucune ontologie. «Ce que l'on choisit comme philosophie dépend de l'homme que l'on est; un système philosophique n'est pas en effet, un instrument mort, ein toter Hausrat, que l'on pourrait prendre ou rejeter selon son bon plaisir; mais il est animé par l'esprit de l'homme qui le possède, beseelt durch die Seele des Menschen der es hat ${ }^{25}$. Je pense que Fichte a raison: le philosophe ne peut se maintenir dans l'innocence épistémologique.

D'où la passion avec laquelle les philosophes défendent leurs idées, si toutefois ils sont philosophes. Le système n'a pas de vie propre indépendamment des options qui le soutiennent. «Pour juger les systèmes philosophiques, les juger comme suffisants, y mettre le sentiment de l'auteur, qui comble tout. $\mathrm{Ne}$ pas les considérer comme pièces, comme étapes, comme idées, comme systèmes, mais comme vies et comme âme... ${ }^{26}$. Pour qu'il en soit autrement, il faudrait que notre spéculation ou bien se soit dépassée dans un savoir absolu, ou bien se soit effondrée dans un nihilisme sceptique. Dans les deux cas, nous en aurions fini avec les croyances et options métaphysiques, mais nous en aurions aussi fini avec le questionnement. ¿a véritable situation du philosophe dans le temps se trouve quelque part entre ces extrêmes et s'il demeure motivé à spéculer, c'est qu'il croit encore à... ou en ...

Considérons cette croyance. Lorsqu'on la compare aux représentations scientifiques et aux systèmes philosophiques, clle se distingue en ceci qu'elle ramène à ses affirmations la réalité ultime et complète: on comprend facilement que deux physiciens s'entendent dans les limites de leur spécialité, même si chacun replace cette même science dans l'englobant de croyances opposées. Ils peuvent s'estimer comme savants tout en constatant leur divergence, par exemple comme chrétien et matérialiste. L'important à remarquer ici, c'est que chacun intègre la science à ses croyances, et non le contraire. Les idées scientifiques et philosophiques se veulent en rapport avec ce qui est, mais elles restent toujours des médiations subordonnées à l'immédiation de la croyance, seule globale, seule compréhensive de la totalité.

25. Erste Einleitung..., paragraphe 5, p. 21.

26. BLONDEL, Maurice, Carnets intimes, tome 1, p. 81. 
Celle-ci n'est pas faite «d'opinions au sujet de...»: elle est adhésion à ce qui est tenu pour la réalité même.

En conséquence, nos croyances peuvent être en grande part, et peut-être même en entier, irréfléchies: elles prennent forme en deça de cette lucidité abstrayante où nous pouvons voir un hiatus entre le contenu et la forme de l'assentiment. Pour les identifier, il faut déblayer l'encombrement de l'érudition, des choses apprises, des opinions, même de la science, pour retrouver le sol de ce sur quoi nous comptons. La croyance n'est pas une noèse thématisante où je prends quelque essence objective en considération, elle est une adhésion où je «compte sur».

Cette adhésion porte sur les choses les plus humbles comme sur les plus hautes, aussi bien sur la chaise à laquelle je me confie que sur Dieu en qui j'espère, aussi bien sur l'amitié de mes intimes que sur le retour de la nuit, quelles que soient par ailleurs mes idées doctrinales au sujet de ces réalités. Ainsi, je pense que ma chaise est un phénomène et non une chose en soi, mais ce n'est pas sur cette vue théorique que je comptc en m'assoyant. J'ai beau m'efforcer à la compatibilité et à la cohérence entre ces deux registres, cela ne supprime pas leur dualité. Cela n'a pas de sens de dire que je crois en une théorie, même s'il est possible qu'une théorie modifie l'ordre de ce en quoi je crois. C'est pourquoi les systèmes de connaissance, même les plus consistants et les mieux fondés, revêtent ce que Ortega y Gasset appelle «a spectral unreal and imaginary character, not really serious ${ }^{27}$. La théorie platonicienne des idées, c'est une chose; la foi de Platon dans la réalité que la théorie cherche à signifier, c'est une autre chose.

Je peux acquérir des connaissances, élaborer des théories, défendre des opinions, et tout cela dépend de ma diligence, alors que je dépends moi-même de mes croyances. En tant que discours et systèmes conceptuels, les philosophies appartiennent au monde de la fantaisie. Placée devant les grandes options métaphysiques, la réflexion critique n'est nécessitće par aucune. Pour emprunter une belle expression de Fichte, devant les possibilités de l'idée, «man sollte mitten inne schweben», c'est-à-dire que

27. Op. cit., p. 299. 
le philosophe en tant que tel maintient ouvertes toutes les possibilités de l'idée en se tenant au milieu, en dedans, comme suspendu en vol. Et il ajoute que cette attitude philosophique est affaire d'imagination créatrice: «Dies ist nun das Geschäft der schaffenden Einbildungskraft. ${ }^{28}$ En arrachant la réflexion à son flottement, le prononcé sur la vérité marque le passage, par la liberté, de l'acte critique à la foi.

Pour mettre en évidence ce décalage entre la fantaisie philosophique que l'on pense et la foi dont on vit, Kant propose un test «amusant ${ }^{29}$. Si le philosophe est le moindrement sincère, peut-être risquera-t-il quelques ducats sur la vérité de sa philosophie, mais s'il s'agit de toute sa fortune, plus il est riche, plus il sera tenté de reconsidérer et d'examiner s'il ne s'est pas trompé, tant il est vrai que ce sont les options pratiques qui tirent à soi les systèmes pensés. Le philosophe hésitant à miser ses ducats sur sa philosophie, c'est bien l'homme revenu à ses croyances et jugeant de là ses fantaisies intellectuelles.

Les jongleries philosophiques seraient un amusement sans douleur tant qu'on n'aurait pas à les souder à ce sur quoi l'on compte effectivement. C'est alors qu'il y a problème de vérité. Dans le libre jeu de l'imagination fichtéenne, le philosophe se meut purement et simplement dans l'ordre des nécessités hypothétiques, et cela lui donne le loisir de débattre de toutes les questions avec des penseurs de toutes options. L'extension de la communication ainsi gagnée n'est pas à dédaigner, mais il faut bien se rendre compte que dans ces limites, le problème de la vérité est ajourné. Sans doute s'occupe-ton d'une certaine vérité dans cette pensée hypothétique, mais au plan logique du pensable, au plan des essences, celles-ci étant apodictiques, dans leur concept et leur idée, et simplement problématiques dans leur être. Ce logicisme s'accommode du pluralisme des ontologies, mais il gêne aussi l'expansion de la pensée dans la mesure où il oblige à passer sous silence ce qui, traditionnellement, constituait l'ambition des philosophies, à savoir l'aptitude du système à se transcender dans un assentiment à l'être. On peut côtoyer des collègues pendant des années, apprécier ce

28. Grundlage..., dritter Teil, no 5, p. 201.

29. Critique de la raison pure, édition Tremesaygues et Pacaud, p. 554. 
qu'ils sont au plan de la suspension critique, sans jamais savoir au juste ce qu'ils sont au plan de l'assertion ontologique.

On l'a suggéré plus haut: le philosophe ne peut ni se retirer dans un scepticisme incapable de faire confiance à aucun jugement, ni s'élever à un savoir qui ne laisserait plus rien à la foi. D'un tel savoir, nous avons l'idée, au sens kantien, et la rupture de la foi naïve, qui marque le début du questionnement philosophique, se situe justement là où l'assentiment de la foi, prenant conscience qu'il n'est pas savoir effectif, se demande ce qu'il est et ce qui le fonde. Autrement dit, la croyance se découvre comme étant possiblement seulement une idée. À ce moment, le croyant perd son innocence, car il se voit comme non-croyant potentiel. Sur ce point Socrate est coupable, et ses juges ont vu juste sur la question de la possibilité d'athéisme que représentait un citoyen de sa sorte. Pour le croyant coupable d'un questionnement suspensif, l'option n'est plus simplement vécue: elle se dédouble en idée. Du coup, je ne suis plus seulement tenu par ma croyance, mais je suis entré dans un point de vue, celui de la pure possibilité, où j’ai à me décider de tenir ma croyance. Je me découvre comme responsable, comme ayant à être croyant ou non.

Cette nouvelle liberté acquise par rapport à la croyance n'est-elle pas l'un des plus puissants motifs de l'effort critique? Quant à la perplexité, la vacillation et l'angoisse, elles seront acceptées comme les compagnes inévitables de cette liberté où l'on tranche les grandes questions non en sachant, mais en suivant les indices idéaux et les intérêts pratiques. Les idées ouvrent un horizon pour la téléologie des espérances humaines, sans pourtant fournir le fondement d'une synthèse entre cette possibilité pensée apodictiquement et l'existence effectivement crue. À l'intérieur du discours logique je peux discerner les absurdités et incohérences impliquées dans une croyance et cela devrait suffire à éliminer ce qui, dans ce système de croyance, est devenu incroyable. Quant à ce qui demeure croyable après le passage de la critique, cela reste comme simple théorème d'une ontologie possible et il faut une nouvelle démarche, une nouvelle décision pour passer de cette ontologie possible à celle de l'assentiment effectif.

La suspension de la croyance par la réflexion critique n'est 
pas terminale, comme si on pouvait se tenir en l'air tout seul après avoir utilisé l'escabeau pour y monter d'abord. Il est inévitable qu'après avoir usé de la liberté pour rompre l'emprise de la croyance, on en use encore pour revenir au sol de l'option et de l'assentiment. Thalès, suspendu à la contemplation des astres, tombe dans un puits, et la légende le mentionne. Thalès, dans la pratique quotidienne, sait très bien diriger ses pas, et la légende ne le mentionne pas. Ainsi l'histoire de la philosophie retient plus volontiers des philosophes leur suspension dans la fantaisie spéculative que leurs croyances effectives. Le plus important échappe, à savoir ce sur quoi le philosophe compte, ce en quoi il met son espérance, ce qu'il attend de l'existence. Tout cela peut bien s'accorder avec le système. Le rapport n'en est pas moins synthétique et n'est pas une pièce du savoir.

N'était-ce pas le tort du dogmatisme d'avoir télescopé ces deux instances? Dans la deuxième préface de la Critique de la raison pure, Kant distingue laborieusement une métaphysique spéculative, (qui sera celle d'une science de la nature), d'une métaphysique pratique. La spéculative est hypothétique, c'est-àdire valide à la condition que des contenus soient donnés a posteriori. La pratique est directement assertorique. Les deux ont les mêmes conditions transcendantales. Alors que, dans l'usage spéculatif, l'idée est régulative d'une représentation qui peut se constituer en science, dans l'usage pratique elle devient permissibilité d'une option ou ingrédient intellectuel d'une décision par laquelle la liberté, transgressant les limites de cette idće sur laquelle elle s'appuie pourtant, opte pour un contenu d'être adéquat à l'exigence formelle de l'idée. En cessant de se prendre pour un savoir, la métaphysique demeure comme horizon de la réalisation humaine. Certes, l'intérêt pour un savoir adéquat aux idćes reste intact, mais il est ajourné dans l'attente de ce qui ne peut se révéler qu'à même la réalisation effective de la destinće humaine.

Cette métaphysique de la raison pratique a semblé trop modeste à l'idéalisme qui n'a pas renoncé à trouver aux idées un contenu spéculativement déterminable. En conséquence, au cours du XIXe siècle, les philosophes n'ont pas cessé de proposer des contenus matériels pour le formalisme kantien, avec ce résultat qu'au lieu de définir l'essence de l'homme d'après les idées, on 
a essayé de mesurer les idées sur l'histoire, sur la nature, sur la transcendance de l'espèce ou de la société par rapport à l'individu, plus récemment sur la transcendance du langage. Ces efforts pour rapaitier l'essence de l'homme dans l'immanence de la nature, de l'histoire, de la temporalité mondaine, de la conscience collective, dans le système des signes, etc, contraignent les idées à venir se soumettre à l'intuition, (au sens kantien), donc aux formes a priori de la sensibilité. Dans les avatars phénoménologiques de cette suite historique, la réalité intégrale, sommée de se dévoiler dans la temporalité, est ainsi déclarée coïncidente avec ce qui est immanent à l'entendement historique.

En somme, depuis Kant, on s'est surtout employé à fermer ce qu'il avait pris soin de laisser ouvert et à ramener l'espoir des hommes dans les limites de ce qui peut se savoir dans le temps. Le dogmatisme commettait l'erreur de subsumer les idées de la raison sous les concepts de l'entendement. Les immanentismes postkantiens ont fait pis encore en voulant forcer les exigences rationnelles à se soumettre à la forme de la temporalité, ajoutant ainsi une illusion intuitionniste à l'illusion transcendantale déjà dénoncée par la critique. La nouvelle dialectique de la raison qu'engendre cette illusion s'exprime dans les philosophies de l'absurde et de l'échec dont notre temps a le secret, et qui sont des immanentismes «spéculaires», car elles fixent à l'être des bornes là où passent les limites de la phénoménalité. Or une telle fixation n'est pas fondée sur des évidences spéculatives: c'est une option, c'est une croyance, tout comme l'affirmation d'une destinće transcendante à l'histoire est, elle aussi, croyance.

Si donc la réflexion philosophique n'est pas elle-même une prise de position effective, elle met toutefois en lumière le fait que nul n'échappe à l'option, le fait que tous tranchent les grandes questions métaphysiques, avec ou sans l'aide d'une philosophie explicite. Autrement dit, le passage par la suspension critique à partir d'une rupture de la croyance ne conduit pas à un état supérieur où la connaissance remplacerait l'option et dispenserait de résoudre le problème de l'existence par un engagement pratique de la liberté. Au contraire, le philosophe se voit ramené au point de départ. «La théorie complète, dit Blondel, conduit forcément à la pratique, puisque le problème 
que l'action a posé, l'action seule peut le résoudre. ...Connaître ne dispense pas de faire; faire peut dispenser de connaître. Si la vie est une énigme même pour les habiles, il suffit d'une entière bonne volonté pour que la lisent couramment même les simples. Peu importe en effet, qu'ils sachent ce qu'ils font pourvu qu'ils fassent ce qu'ils savent: la sincérité de la conscience et le discernement des vérités vivifiantes tiennent plus aux actes qu'aux pensées; car jamais il ne suffit entièrement de faire ce qu'on croit bon, il faut encore qu'il soit bon de croire qu'on doit le faire. La logique de l'action cherche uniquement à découvrir un itinéraire qui permette à l'intelligence des doctes de rejoindre lentement et sûrement les hauteurs des humbles et des petits; elle les amène à un point de départ. Mais la lumière dont elle éclaire la route ne dispense personne de l'effort qui reste nécessaire pour y monter. ${ }^{30}$

La foi ontologique alimente directement la pratique, sans attendre la réflexion critique. Cette pratique, qui a sa propre logique, est «la source alimentant la réflexion, la relançant toujours $\gg$. Le discours philosophique reprend dans son ordre propre, sous forme de thématisation formelle, cette logique déjà immanente à l'assentiment et à l'action. C'est pourquoi la réflexion n'a pas à craindre «que son mouvement s'arrête, par épuisement, ni que son champ se rétrécisse», car elle revient sur une ontogénie qui ne peut elle-même s'arrêter ${ }^{31}$. Il n'y a pas à craindre non plus que le rationalisme de la réflexion conduise au dessèchement de la cordialité, si l'on se souvient que le sentiment ne se déduit pas de la philosophie mais vit en symbiose avec la croyance. Il appartient à la réglexion critique de seulement lire ce que l'action contient, de discerner ce qu'il y a d'onto-logique immanent à l'onto- génique ${ }^{32}$.

30. L'Action, essai d'une critique de la vie et d'une science de la pratique, Paris, Alcan, 1893, p. 474.

31. MANSIR, Jean « Réflexions sur les conditions d'une critique philosophique de la foi », dans Revue des Sciences Philosopbiques et Théologiques, 51 (1967) 173.

32. Tout en admettant que le dévoilement de l'être puisse correctement s'appeler «ontologie», Blondel employait le terme «ontogénie» pour signifier la solidarité de ce dévoilement avec l'ontogénèse de l'homme. La philosophie de l'être passe par la médiation de l'homme espérant, et agissant pour égaler à son espérance. - L'Être et les êtres, Paris, Alcan, 1935 , p. 234. 
La philosophie n'est donc nécessaire que pour démontrer sa non-nécessité. «La philosophie est nécessaire pour établir qu’elle n'est pas suffisante, et que rien de scientifique ou de rationnel ne satisfait le besoin de savoir et n'égale le pouvoir ou l'ambition d'être. ${ }^{33}$ » L'acharnement des philosophes serait inexplicable sans l'attrait de cet espace intelligible où l'existence est en suspens, où l'on dispose d'un appui à partir duquel le consentement à l'être se manifeste comme second par rapport à la simple possibilité. En grattant son papier, voilà que le philosophe s'imagine au poste de commande, où l'on décide du meilleur des mondes, comme le Dieu de Leibniz. Ce rêve intellectuel de la volonté de puissance se déroule dans la logique, dans la transcendance d'un discours universel. En disant le mot, le philosophe est donc supprimé comme instance disante par le mot. D'une part, le philosophe voudrait que l'intégrale réalité soit comme elle paraît rien qu'à y penser, car il pense que la pensée est au pouvoir de la pensée. D'autre part, il disparaît dans ce qu'il pense, si son vouloir aboutit. Il n'y a pas de place pour monsieur Friedrich Hegel dans l'hégélianisme. Kierkegaard lui cherche querelle sur ce point ${ }^{36}$, et je plaide pour son parti. Évidemment, si la logique de Hegel était équivalente à l'ontologie vraie, il n'y a plus de monsieur Friedrich: si ma logique est l'ontologie, je suis Dieu. Qu'on me permette de me récuser.

«La réflexion n'est pas une possession effective, mais un projet de possession ou une domination formelle. Et c'est précisément cette non-possession de nous-mêmes, cette privation d'intuition immanente à la vie qui fait qu'en dépit de la foncière unité du composé bumain suivant laquelle la vie ne s'organise que par la pensée et la pensće ne se réalise que par la vie, l'être vivant et le sujet pensant demeurent en nous extérieurs l'un à l'autre; de sorte que la vie apparaît à la pensće comme une force aveugle qui l'opprime, et qu'ensuite, cherchant à se saisir elle-même, la pensée ne s'apparaît plus que comme une forme idéale, sans vie et sans réalité ${ }^{35}$.

Dans la mesure où je m'identifie à la fois avec la formali-

33. Carnets..., tome 2, p. 181.

34. Post-Scriptum aux Miettes pbilosopbiques, traduction Paul Pctit, Paris, Gallimard, 1949, pp. 72-83.

35. PALIARD, Jacques, Intuition et réflexion, Paris, Alcan, 1925, p. 204. 
sation réflexive et la croyance effective, ma réalité propre ne se définit ni par le vécu, qui me paraît d'abord être un agglomérat factice, «un concours d'accidents subis», ni par la destinée idéale, mais simplement possible, dont je me parle dans une philosophie transcendantale. Quelle est donc la modification introduite dans l'existence par la réflexion critique? C'est la connaissance de l'inadéquation du vécu à l'exigence de l'idée et de l'inadéquation de l'idée à ce que j'attends de l'existence. Je me serais donc perdu dans mes cogitations et je me chercherais au-delà dans une réalisation qui transcende l'idée pourtant reconnue comme normative. Dans l'un et l'autre mouvement il y a un «leap of faith ${ }^{36}$, une option, un «fait» de liberté. $\bar{A}$ la fois je me perds dans mes idées, et je perds mes idées pour m'emparer de ce qu'elles signifient sans le donner, ... ou pour le recevoir, s'il est vrai que l'accomplissement est grâce et salut. "Comme la marche qui n'est qu'une chute arrêtée, la pensée s'appuie sur ce qui suit ... ${ }^{3 \top}$.

Dans cette instabilité entre l'immédiat de la croyance et la suspension réflexive, le philosophe oscillant paraît tricoter sa marche comme un homme ivre parmi un peuple à jeun, mais lui, il entretient la secrète prétention d'être seul éveillé parmi des endormis. Le sympathique compilateur Diogène Laërce nous transmet ce qu'il a lu dans les vieux livres, à savoir que l'Etna a recraché une des sandales d'Empédocle, intacte. Ce mythe m'est un sujet de rêverie sans fin. Diogène dit qu'elle était de bronze et j'imagine son éclat métallique et fauve, au petit matin, sur le bord du cratère. C'est la sandale du discours, de la logique, de la nécessité objective, de la possibilité. Enpédocle n'a pu l'emporter dáns l'autre monde, pas plus qu'on n'y emporte l'argent. Mais je me réconforte à penser qu'elle lui a servi jusqu'atı bout. Pour le reste, je ne sais rien, sinon qu'il a passć la porte de l'éternité en claudiquant dans la sandale de la décision et de la liberté. Du cratère ne sortent que des fumerolles sulfureuses, signes des fusions ignées au sein de l'abime, dont nous ne voyons rien.

36. HINNERS, Richard C., Ideology and Analysis, a Rebabilitation of Mctaphysical Ontology, Bruges-New York, Desclée de Brouwer, 1966, p. 51. (Distribution au Canada: Les Éditions Bellarmin, Montréal.)

37. Carnets..., tome 2, p. 176. 